\title{
When is EROI Not EROI?
}

\author{
Michael Carbajales-Dale ${ }^{1} \mathbb{D}$
}

Received: 5 November 2019 / Revised: 25 November 2019 / Accepted: 27 November 2019 / Published online: 3 December 2019 (c) The Author(s) 2019

\begin{abstract}
This paper outlines some very real issues with the use of energy return on investment (EROI) for comparing different energy delivery pathways, particularly when directly comparing EROI calculated at the scale of a single energy facility (as a ratio of full lifetime energy transfers) with that calculated at the scale of a geographical region or industry (as a ratio of annual energy flows). While these two ratios may converge, it is only under a very specific set of circumstances. The aim of this paper is to outline this issue in detail and provide some specific examples of the difference between these two ratios for the global wind and photovoltaics industries.
\end{abstract}

Keywords Net energy analysis · Energy return on investment (EROI) · Renewable energy · Solar energy $\cdot$ Life cycle assessment $\cdot$ Energy systems analysis

\section{Introduction}

In a recent paper, Brockway et al. highlight 'apples and oranges' nature of comparing the energy return on investment (EROI) of oil at the wellhead with electricity production from renewable technologies (Brockway et al. 2019). Clearly this is not a fair comparison, just as we would not directly compare the price of oil, or perhaps coal, with the price of electricity. There is a good reason why society is willing to exchange around three units of solid, liquid, or gas fuel for one unit of electricity output. Electricity is easier to transport and much more useful (i.e., can be put to a wider variety of end uses) than the fuel from which it is often made, though of course has problems when we try to store it (more on this later). As such, Brockway et al. calculate the EROI using annual industry data from IEA and input-output models to extend the oil delivery pathway to include transmission and conversion to electricity, since this is often not done (at least in the US, with only $1 \%$ of oil going toward electricity) perhaps a fairer comparison might

Electronic supplementary material The online version of this article (https://doi.org/10.1007/s41247-019-0065-8) contains supplementary material, which is available to authorized users.

Michael Carbajales-Dale

madale@clemson.edu

1 Environmental Engineering and Earth Sciences, Clemson University, Clemson, SC, USA be calculating the EROI when comparing the energy service of an internal combustion vehicle fueled by oil-derived gasoline with a battery-electric vehicle powered by electricity generated from renewable resources. Whatever the case, the electric-to-electric comparison of EROI is far fairer than the liquid-fuel-to-electricity comparison that is common.

However, in their Table 1, Brockway et al. directly compare this new EROI ratio with that of renewable electricity generation from Raugei et al. (2012). Unfortunately, however, these two numbers are not directly comparable either, as will be explained in the rest of the paper. We will argue that a better term for the industry-scale ratio is power return on investment (PROI), as used by (Murphy et al. 2016; Leccisi et al. 2018). A value of PROI for the global wind and solar photovoltaic (PV) industries will be presented.

\section{Set the Scale: EROI Versus PROI}

As has often been commented, EROI is very easy to define, as in:

EROI $=\frac{\text { Energy output }}{\text { Energy investments }}$

Unfortunately, as defined, the energy outputs and investments do not have any time period associated with them; the EROI could be calculated over the whole lifetime of a facility, or over just 1 year. From the perspective of a single 
facility, it makes little sense to choose a single year for the time period as during construction, before the plant comes online (and after shutdown), the EROI will be zero. The returns on the investment in construction do not happen in the current time period, but will happen (maybe some years) in the future. Only during operation, would this EROI have some non-zero value. As such, at the scale of a single facility, it makes sense to set the time period of analysis as the lifetime of the facility and as such, the numerator and denominator are both total energy transfers of energy integrated over the whole period. We have bounded the system boundary in time to account all energy flows into and out of the system. If the system boundary is extended either forward or backward in time, it will not change the results of the analysis, since there are no flows that are not already captured.

What about at the scale of an industry? Well, here we don't have an analog to the facility lifetime and we are certainly not thinking about the lifetime of the whole industry. We are also more justified in choosing an annual time period, since the investments and outputs are more comparable. As such, the numerator and denominator are energy flow rates (energy per year, i.e., power) in a year, not cumulated over multiple time periods. The system boundary in time (i.e., each year) is not sufficient to account all energy flows into and out of the system. Output in the current time period is stemming from investments in previous periods and investments in this period will be realized through energy output in future periods, such that the problems should approximately 'come out in the wash'.

However, notice that this is not necessarily the case. The solar and wind industries are growing rapidly, such that current investment is not matched by current output from the industry. Even mature industries, such as oil and gas, can see large shifts year-to-year in drilling activity, causing 'EROI' (actually PROI) to bounce up and down, as can be seen in Fig. 3 of Brockway et al. (2019) and more explicitly in Fig. 3 of Guilford et al. (2011).

When we think of EROI calculation for wind or solar, we probably think of an analysis conducted at the scale of a single installation, e.g., a wind or solar farm (Huang et al. 2017). Although some industry- or regional-scale analyses have been conducted (Dale and Benson 2013; CarbajalesDale et al. 2014), this is not the norm. One notable exception is the analysis of the Spanish PV industry by Prieto and Hall (2013). In fact, this facility-scale analysis is generally true of most EROI of electricity generation, being conducted for a single power plant. However, the opposite is normally true for oil and gas. Most (if not all) 'EROI' calculations are looking at the whole industry, or at least production within a region (Brandt 2011; Brandt et al. 2013). Indeed, this is the case for Brockway et al. using industry-scale data. Confusingly, the term EROI is used to refer to both. Here we run into issues however, since the two 'EROIs' are not directly comparable.

At the scale of a single facility, we can distinguish three distinct, consecutive phases: construction, operation, and decommission. We can define the EROI as the ratio of outputs to investments over these three phases in the life cycle of the facility. A region or industry, however, is composed of multiple, overlapping projects engaged in a continual process of construction, operation, and decommission all at the same time, such that there is no analog to the life cycle for the facility. As such, this ratio is calculated using (normally annual) energy flows (i.e., power), hence we use the term power return on investment (PROI) after (Murphy et al. 2016; Leccisi et al. 2018) but the more general term power return ratio (PRR) has also been used (King et al. 2015). Many studies refer to this PROI ratio as EROI and compare it directly with values calculated at the facility scale. Indeed, Brockway et al. make such a comparison in their Table 1 comparing EROI from different studies.

The true relationship between facility-scale EROI and the PROI of a region or industry composed of such projects is complex; involving when in the project lifecycle the investments are made and whether the industry is growing or at steady-state. This relationship is derived in the electronic supplementary information. From Supplementary Materials Eq. 14 we can see that regional-scale PROI approaches facility-scale EROI only as industry growth rate approaches zero, i.e., at steady-state, and as both the installation time and decommissioning time approach 1 year. For example, imagine two different industries, both growing at $10 \%$ per year, both composed of projects with a lifetime of 20 years and facility-scale EROI of 10. For one industry (let's call it non-renewable), the energy investments all come during the operation phase such that each year, the energy investments are one tenth of the energy output. This obviously gives us a PROI in each year of 10 .

For the other industry (let's call it the renewable industry), the energy investments all come at the start of each project during the construction phase but there are zero on-going operating costs. In this case, the annual PROI in each year is around 2.9. This will continue while the industry continues to grow at that rate. Should the industry stop growing, to remain at steady-state (i.e., additions just replacing decommissions), only then will the PROI approach 10 . Interestingly, this essentially replicates the result of Prieto and Hall for the Spanish solar PV industry where they used a facility-scale EROI of around 8 but calculated an industryscale 'EROI' (actually PROI) of around 2.4 (Prieto and Hall 2013).

In more detail, we can take historic data on the installed capacity and market share of the PV and wind industries (EIA 2019; Faunhofer 2019; GWEC 2019; IEA 2019; IRENA 2019; Europe 2018; UN 2019; WWEA 2019), 


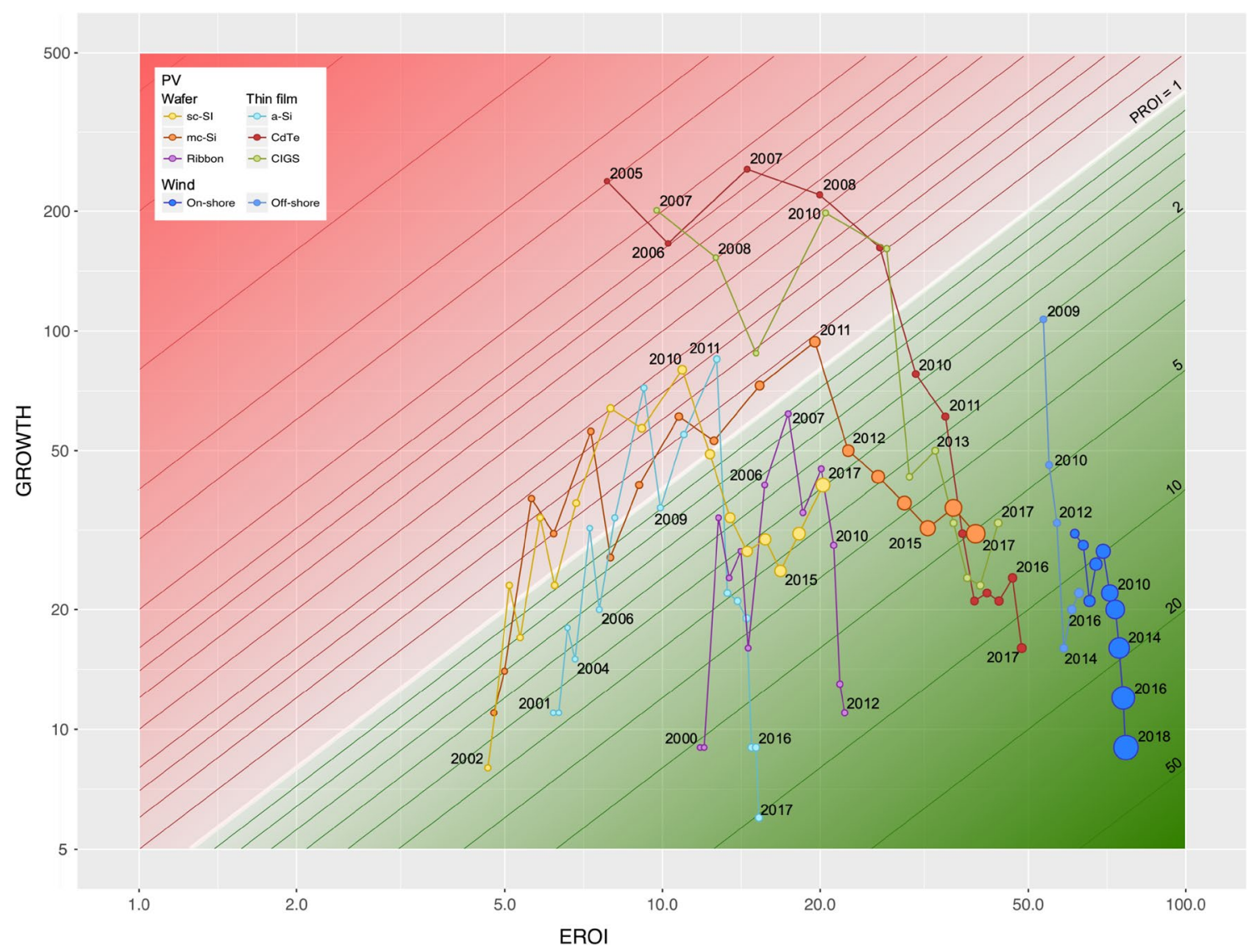

Fig. 1 PROI of global PV and wind industry technologies as a function of facility-scale EROI and growth rate [\% per year]. The size of the bubbles represents the cumulative installed capacity for each technology. The main PV technologies are single crystalline (sc), multicrystalline (mc), ribbon, and amorphous (a) silicon ( $\mathrm{Si}$ ), cadmium tel-

combined with learning rates on energy investments into these technologies (Carbajales-Dale et al. 2014; Dale and Benson 2013) to track regional-scale PROI as a function of facility-scale EROI (using primary energy equivalent for the electricity output) and industry growth rate (in percent per year), to create Fig. 1. As can be seen, on-shore wind in 2018 had a facility-scale EROI of over 70, but due to industry growth, the regional-scale PROI was lower at around 25. In a more extreme situation, the facility-scale EROI of cadmium telluride (CdTe) in 2005 was fairly respectable at around 8 , but due to the incredibly rapid growth rate of over $200 \%$ per year, the regional-scale PROI was 0.1 , i.e., the technology required more energy than was being supplied by panels in place.

For both industries as a whole in 2018, regional-scale PROI for wind is around 23, despite new capacity additions having a facility-scale EROI of close to 80. For PV, the composite facility-scale EROI of the different technologies was over 30 , but the regional-scale PROI is below luride (CdTe), and copper indium gallium (di)selenide (CIGS). Wind is split between on-shore and off-shore. We assume an average capacity factor of $11.5 \%$ for PV (global average is actually closer to $15 \%$ ) and $23 \%$ for wind, that all technologies have a 25 year lifetime, and take 1 year to construct. We assume no operational investments

5. In 2012, the year of Raugei et al.'s analysis, the PV industry growth rate was $42 \%$, meaning that although the composite facility-scale EROI was around 19 (reported as 19-38 in Table 1 of Brockway et al. but in reality, it is skewed toward the more energy intensive crystalline silicon technologies that dominate(d) the market), the regional-scale PROI was below 2 . If we do not adjust the electricity output for primary energy equivalence, these values drop by two-thirds to 6 and 0.6 respectively. These values are more appropriate as comparisons for Brockway et al.

As such, we should be very careful in directly comparing EROI with PROI, since in so doing, we are implicitly assuming that the industry or region is operating at steady-state. Clearly this is not currently the case for the wind and solar industry which both have installed capacity growth rates of over $10 \%$ per year. In fact, the oil and gas industry is also not in operating at steady-state. Estimates of 'EROI' (actually PROI) for US oil and gas production show a strong negative correlation to drilling intensity 
(Guilford et al. 2011), i.e., as drilling increases in any 1 year, the PROI of oil production in that year decreases. From a philosophical perspective too, there should be concerns at any activity that assumes steady-state, since one of the primary motivators for considering EROI is to understand transitions in the energy sector.

\section{Conclusion}

In summary then, congratulations are due again to Brockway et al. for a fine paper highlighting an important issue in comparison of EROI results. The purpose of this paper was to highlight another methodological issue, that of scale of the analysis (facility vs. region) which must also be accounted, leading to the calculation of two incomparable energy return ratios, EROI and PROI, respectively.

Open Access This article is distributed under the terms of the Creative Commons Attribution 4.0 International License (http://creativeco mmons.org/licenses/by/4.0/), which permits unrestricted use, distribution, and reproduction in any medium, provided you give appropriate credit to the original author(s) and the source, provide a link to the Creative Commons license, and indicate if changes were made.

\section{References}

Brandt AR (2011) The effects of oil depletion on the energy efficiency of oil production: bottom-up estimates from the California oil industry. Sustainability 3:1833-1854

Brandt AR, Englander J, Bharadwaj S (2013) The energy efficiency of oil sands extraction: energy return ratios from 1970 to 2010 . Energy 55:693-702

Brockway PE, Owen A, Brand-Correa LI, Hardt L (2019) Estimation of global final-stage energy-return-on-investment for fossil fuels with comparison to renewable energy sources. Nat. Energy 4:612-621

Carbajales-Dale M, Barnhart CJ, Benson SM (2014) Can we afford storage? A dynamic net energy analysis of renewable electricity generation supported by energy storage. Energy Environ Sci 7(5):1538-1544

Dale M, Benson SM (2013a) Energy balance of the global photovoltaic (PV) industry-is the PV industry a net electricity producer? Environ Sci Technol 47(7):3482-3489
Dale M, Benson SM (2013b) Energy balance of the global photovoltaic (PV) industry-is the PV industry a net electricity producer? Environ Sci Technol 47:2489-3428

EIA (2019) International Energy Statistics. US Energy Information Administration, Washington, DC. https://www.eia.gov/beta/inter national/data/browser. Accessed 27 Sept 2019

Europe SolarPower (2018) Global market outlook 2018-2022. Solar Power Europe, Brussels

Faunhofer (2019) Photovoltaics report 2018

Guilford MC, Hall CAS, O'Connor P, Cleveland CJ (2011) A new long term assessment of energy return on investment (EROI) for U.S. oil and gas discovery and production. Sustainability 3(10): $1866-1887$

GWEC (2019) Global wind report 2018

Huang YF, Gan XJ, Te Chiueh P (2017) Life cycle assessment and net energy analysis of offshore wind power systems. Renew Energy 102:98-106

IEA PVPS (2019) Snapshot of global PV markets 2019

IRENA (2019) Renewable capacity statistics 2019

King C, Maxwell J, Donovan A (2015) Comparing world economic and net energy metrics, part 1: single technology and commodity perspective. Energies 8(11):12949-12974

Leccisi E, Raugei M, Fthenakis V (2018) The energy performance of potential scenarios with large-scale PV deployment in Chile-a dynamic analysis. In: 2018 IEEE 7th world conference on photovoltaic energy conversion (WCPEC) (a joint conference of 45th IEEE PVSC, 28th PVSEC \& 34th EU PVSEC), pp 2441-2446

Murphy DJ, Carbajales-Dale M, Moeller D (2016) Comparing apples to apples: why the net energy analysis community needs to adopt the life-cycle analysis framework. Energies 9(11):1-15

Prieto PA, Hall CAS (2013) Calculating the Energy Return on Energy Invested (EROEI or EROI) for Spain's solar photovoltaic energy. Springer, New York

Raugei M, Fullana-i-Palmer P, Fthenakis V (2012) The energy return on energy investment (EROI) of photovoltaics: methodology and comparisons with fossil fuel life cycles. Energy Policy 45:576-582

UN (2019) UN energy statistics database. http://data.un.org/Brows e.aspx?d=EDATA. Accessed 27 Sept 2019

WWEA (2019) Global total installed capacity. Wind Energy International. https://library.wwindea.org/global-statitistics-1980/. Accessed 27 Sept 2019

Publisher's Note Springer Nature remains neutral with regard to jurisdictional claims in published maps and institutional affiliations. 